Témoigner Témoigner. Entre histoire et mémoire

Getuigen Revue pluridisciplinaire de la Fondation Auschwitz

$121 \mid 2015$

Violences radicales en scène

\title{
Revisiter l'Histoire en déconstruisant les événements
}

Entretien avec Guy Cassiers

Interview met Guy Cassiers. Deconstructie van het verleden, een nieuwe kijk op

de geschiedenis

\section{Guy Cassiers et Edwige Perrot}

\section{(2) OpenEdition}

\section{Journals}

Édition électronique

URL : https://journals.openedition.org/temoigner/3279

DOI : 10.4000/temoigner.3279

ISSN : 2506-6390

Traduction(s) :

Interview met Guy Cassiers - URL : https://journals.openedition.org/temoigner/3293 [nl]

Éditeur :

Éditions du Centre d'études et de documentation Mémoire d'Auschwitz, Éditions Kimé

Édition imprimée

Date de publication : 1 octobre 2015

Pagination : 63-68

ISSN : 2031-4183

Référence électronique

Guy Cassiers et Edwige Perrot, «Revisiter l'Histoire en déconstruisant les événements », Témoigner. Entre histoire et mémoire [En ligne], 121 | 2015, mis en ligne le 01 octobre 2016, consulté le 04 février 2022. URL : http://journals.openedition.org/temoigner/3279 ; DOI : https://doi.org/10.4000/temoigner. 3279 


\section{Revisiter l'Histoire en déconstruisant les événements}

$\rightarrow$ Entretien mené par Edwige Perrot le 17 Juin 2015

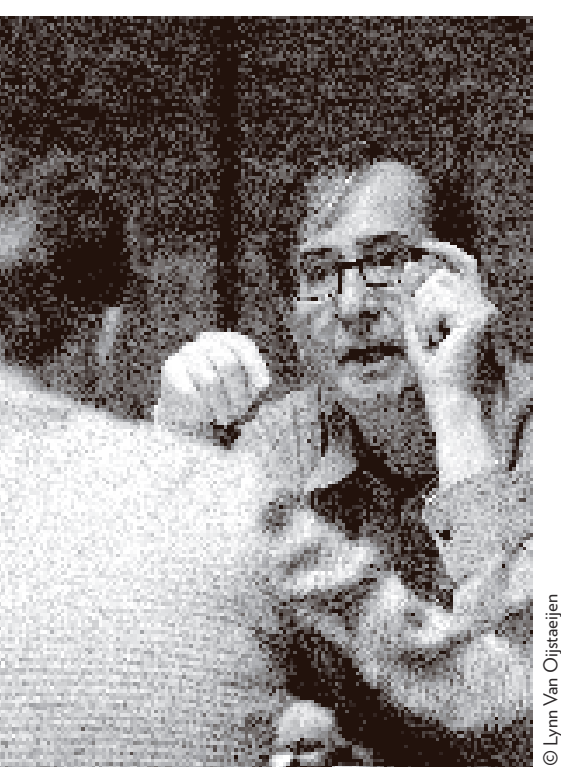

La violence politique est un thème récurrent dans l'ensemble de votre œuvre : Rouge décanté, Mefisto for ever, Wolfskers, Atropa, Cœur ténébreux, Les Bienveillantes bientôt. Pourquoi est-ce si important pour vous d'aborder ce sujet à travers vos mises en scène?

Guy Cassiers : Le plus bel exemple n'est pas dans cette liste et remonte à une vingtaine d'années (1995). Il s'agit de Time's Arrow de Martin Amis. Pour moi, la manière dont Amis raconte l'histoire est très importante par rapport aux thèmes du pouvoir, de la mémoire, de l'importance de se souvenir de l'Histoire et de ce que l'on peut en faire. Dans Time's Arrow, Martin Amis raconte l'histoire d'un homme selon un récit construit à rebours. L'histoire commence à sa mort, au moment où les électrochocs prodigués par les médecins pour le réanimer vont en fait réveiller sa conscience. À partir de là, le personnage va remonter le cours de son histoire, instant après instant, comme si les événements de sa vie se rembobinaient les uns après les autres dans les moindres détails. Et cette conscience, ne comprenant ni ce qui lui arrive ni où ça la mène, va chercher à comprendre le mouvement dans lequel elle est prise. Or, pour moi, la partie la plus frappante du livre et du spectacle que nous en avons fait, est que cette vie commence à faire sens pendant la Seconde Guerre mondiale, lorsque le personnage est un médecin à Auschwitz. Comme chaque action est présentée à rebours, on voit apparaître des hommes et des femmes à partir des cendres. Puis le médecin leur donne des cheveux, leur met des dents en or. Et à ce moment-là, la conscience réalise que ce retour en arrière prend du sens, explique des choses. Pour moi, ça a été un choc de lire ce que l'on sait sur ce qui s'est passé pendant la Seconde Guerre mondiale en adoptant la perspective différente qu’offre la mise en récit. En prenant l'histoire à rebours, en déconstruisant l'enchaînement des faits, on peut se rapprocher d'une manière plus directe encore, pas seulement des faits, mais de leur signification, du sens de ce qui s'est passé là. Et apprendre d'eux. L'intensité émotionnelle que procure le fait de remonter les événements, des conséquences aux causes, me fait voir davantage la réalité, comment ils se sont produits, leur mécanique. Et cela me rend responsable, en tant qu'être humain, de faire en sorte que ces mécanismes ne se reproduisent plus jamais. Nous devons rester vigilants. Tout le monde dit «Ok. Ce qui s'est passé dans le passé ne se reproduira jamais... » Je pense que tout peut encore arriver. 
Revisiter l'Histoire en déconstruisant les événements (suite)

\section{LE THÉÂTRE COMME BASTION DE RÉSISTANCE À L'OUBLI}

Le théâtre semble être pour vous un lieu de Mémoire... pourtant c'est aussi un espace qui se prête à la fiction...

G.C. : Oui, je pense que c'est très important que nous gardions en mémoire l'Histoire. L'important n'est pas seulement de savoir ce qui s'est passé, mais de susciter chez le spectateur une émotion personnelle qui le relie à ce qui s'est passé. Nous devons rester en lien avec elle. Et considérer notre responsabilité pas seulement à travers le souvenir, le devoir de mémoire, mais aussi à travers l'usage que nous en faisons pour demain. On n'emporte pas l'Histoire avec soi, mais on apprend d'elle pour l'avenir. Et là où le théâtre peut agir, peut nous aider, c'est en suscitant cette intensité émotionnelle qui est liée aux faits historiques, cela même lorsqu'elle passe par la voie de la fiction. D’après moi, nous sommes tellement exposés, quotidiennement, à une surcharge d'images exposant la cruauté partout dans le monde, la destruction, et la souffrance. Mais le problème avec les images c'est qu’à force de les voir, on s'y habitue. On est tellement habitués à voir des choses qui semblent dépasser la réalité, qu’on finit par ne plus les voir et par les oublier. Or, ce que permet l'art, c'est de ne jamais oublier.

\section{COMPRENDRE LES MÉCANISMES DE LA VIOLENCE}

Vous abordez l'Histoire, le pouvoir et ses atrocités en mettant en scène des personnages souvent cruels, qui le sont devenus ou qui le deviennent : un jeune meurtrier dans Butcher Boy, une victime des camps de concentration japonais dans Rouge Décanté, les figures de dictateurs dans Wolfskers, un témoin de l'horreur coloniale dans Cœur ténébreux, et bientôt un intellectuel et bourreau dans les Bienveillantes. Pourquoi est-ce si important pour vous de présenter la violence à travers ces différents prismes?

G.C. : Rouge décanté commence par un homme qui est isolé, qui ne veut pas communiquer et qui dit que sa vie est terminée. Ce qu’il y a de magnifique dans le livre, et que nous avons essayé d'accomplir dans le spectacle, c'est que, même si au début on résiste au personnage, qu'on ne veut absolument pas s'identifier à lui, au fil de son témoignage on finit par se sentir coupable de notre résistance, de l'avoir jugé facilement et hâtivement. Parce qu'aussi antipathique qu'il puisse être, une fois qu'il nous a raconté ce qui lui est arrivé dans son enfance, on commence à comprendre son comportement. Sans pour autant l'excuser de mal agir, il nous amène à nous interroger sur ce que nous aurions fait à sa place, si nous avions vécu la même chose. Pourrions-nous, nous aussi, devenir comme lui dans les mêmes circonstances?

Avec Butcher Boy de Patrick McCabe, c'est un peu la même chose. Il s'agit d'un enfant isolé, issu d'un milieu social et familial peu favorables, et qui ne comprend pas vraiment le monde qui l'entoure. Il travaille dans un abattoir et finit par ne plus voir la différence entre les animaux et les hommes, si bien qu'il va se mettre à tuer. Parce qu'il n'a pas eu l'éducation pour développer l'émotion et la pensée. Dans le spectacle qu'on a créé, un monologue, nous avons voulu montrer comment 


\section{VIOLENCES RADICALES EN SCÈNE}
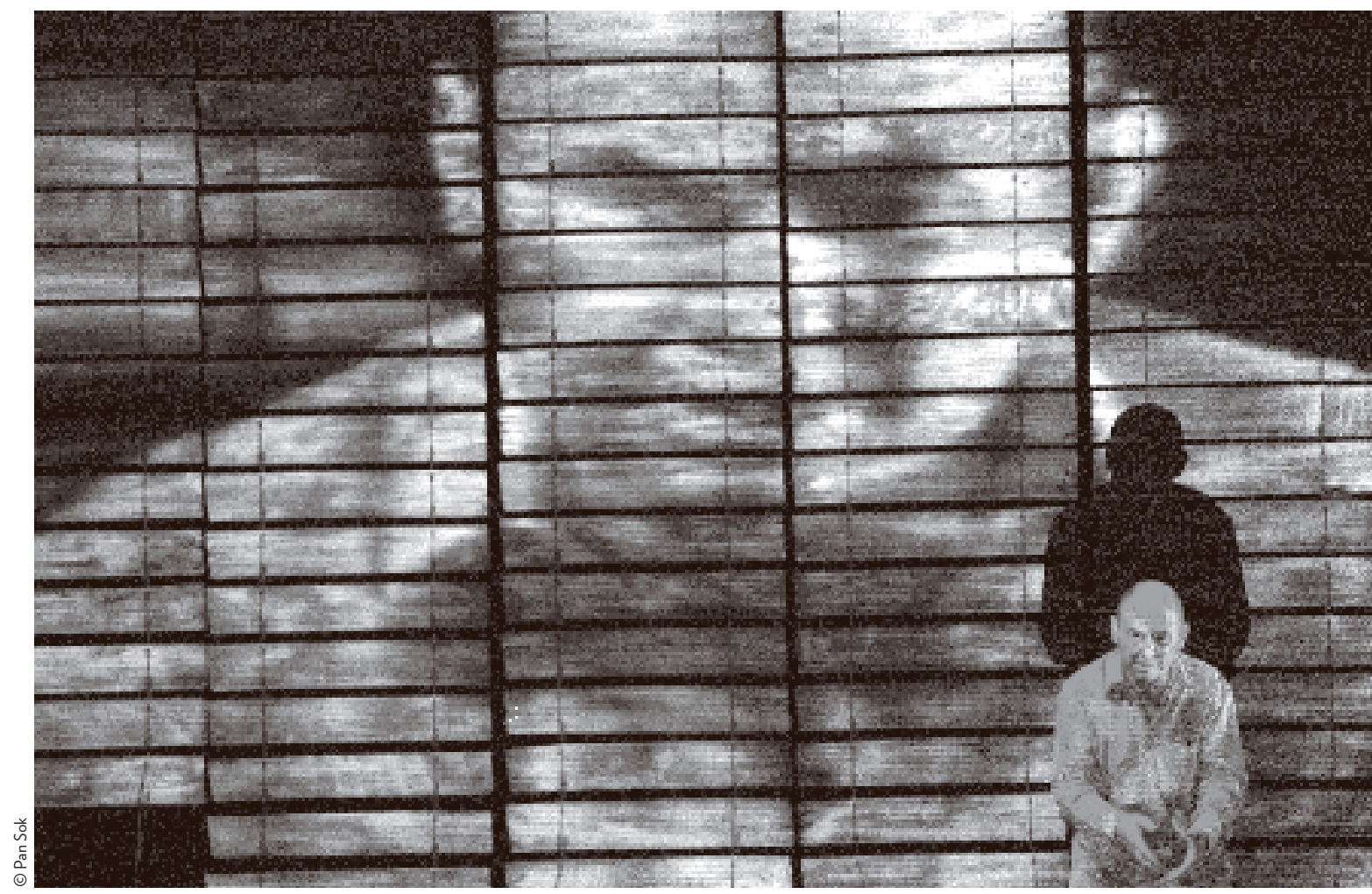

- Rouge décanté.

cet enfant réagissait au monde, comment il le percevait, comment il entendait les autres. La parole des autres personnages passait par de la projection vidéo de leur texte sur scène. Les spectateurs lisaient et, à la manière de réagir de l'acteur, ils pouvaient comprendre l'impact des paroles sur l'enfant, comprendre son univers, et son comportement. Il s'agissait de montrer la distance entre cet enfant et le monde, tenter de comprendre ce personnage et surtout montrer comment une personne peut devenir un meurtrier : décortiquer les mécanismes. C'est pour cela que j’aime mettre en scène des personnages cruels, des personnages qui ont du pouvoir ou qui sont laissés pour compte, ou encore qui ne trouvent pas leur place, et ce, même si leurs actes sont inhumains. Je pense que si l'on comprend comment cela fonctionne, ça peut nous aider à éviter que cela se reproduise.

\section{L'HORREUR À PORTÉE DE TOUS}

Dans Cœur ténébreux, il s'agit d'un personnage qui va à la rencontre d'un homme pour l'aider. Mais au fur et à mesure de son trajet, c'est dans un voyage vers l'horreur qu'il s'engage, au point d'être lui-même gagné par le mal. Dès lors, l'on s'aperçoit qu'en 
Revisiter l'Histoire en déconstruisant les événements (suite)

(1) Susan Sontag, Regarding the pain of others, Penguin books, 2004, p. 106. chacun de nous réside une certaine propension à l'horreur. Et c'est aussi valable pour Les Bienveillantes. Au début, le personnage reconnaît qu'il est le mal, qu'il a fait tout ce qu'une personne ne devrait jamais faire, mais il nous interroge aussi : dans la même situation, auriez-vous agi différemment? Au début, on est certain que oui, ça n'aurait jamais pu nous arriver, mais ce qui est étrange, c'est qu'au fur et à mesure qu'on lit le livre, on commence à douter de nos certitudes, à nous demander si nous aurions fait mieux, si nous n’aurions pas agi comme lui. Il est important de réaliser le danger : cela peut encore arriver!

Est-ce la raison pour laquelle vous montrez à chaque fois ce qui se passe dans l'esprit des personnages, en utilisant la vidéo pour donner à voir leurs monologues intérieurs?

G.C. : Oui. Nous avons besoin de mettre en scène des situations, des personnages, mais au fond ce qui prévaut ce sont les pensées. C'est de cela dont il s'agit. Du comportement humain et de son rapport au langage. Comment celui-ci influence les comportements? Le pouvoir d'une personne ne relève pas seulement de ce qu'elle dit, mais réside dans sa capacité à expliquer joliment ce qu'elle est en train de dire. C'est là que ça se joue : on est tout le temps séduit par le pouvoir du langage, le contenu du discours passe après.

Le langage justement, et le texte tiennent une place prépondérante dans vos spectacles au même titre, d'ailleurs, que l'usage de la vidéo et l'utilisation d'images...

G.C. : Pour moi, ce qui est essentiel au théâtre, c'est qu'on utilise le langage, qu'on lui accorde de l'importance et qu'on questionne la manière dont on l'utilise, dont il est utilisé. Les images peuvent avoir un impact extrêmement fort en extrêmement peu de temps, mais on peut très facilement en être déconnecté. Le langage, d'après moi, travaille de manière beaucoup plus lente, on le digère différemment. Physiologiquement, cela doit surement se jouer à un endroit différent du cerveau. J'apprécie beaucoup ce que Susan Sontag écrit dans l'un de ses essais : "There's nothing wrong with standing back and thinking. To paraphrase several sages: "Nobody can think and hit someone at the same time." "1 C'est une manière de dire que penser peut nous protéger de notre propre cruauté. Ça a l'air simple, mais je pense que c'est là l'essence : ilvaut mieux faire réfléchir et faire discuter les gens. La guerre, la violence, arrivent quand le langage défaillit, échoue. C'est pourquoi il me semble important de développer la qualité du langage et la qualité du raisonnement.

\section{RESTER VIGILANT FACE AU POUVOIR DU LANGAGE DE LÉGITIMER L'INACCEPTABLE}

Vous avez choisi de monter certains spectacles qui abordent la question du pouvoir et son rapport au langage en réponse à la montée des populismes en Flandre et en Europe. Quel rapport faites-vous entre le pouvoir, la violence et le langage?

G.C. : Oui, c'était le cas avec Mefisto for ever, c'est ce que l'on va faire avec les Bienveillantes et ce que l'on fait actuellement avec Le sec et l'humide de Jonathan 


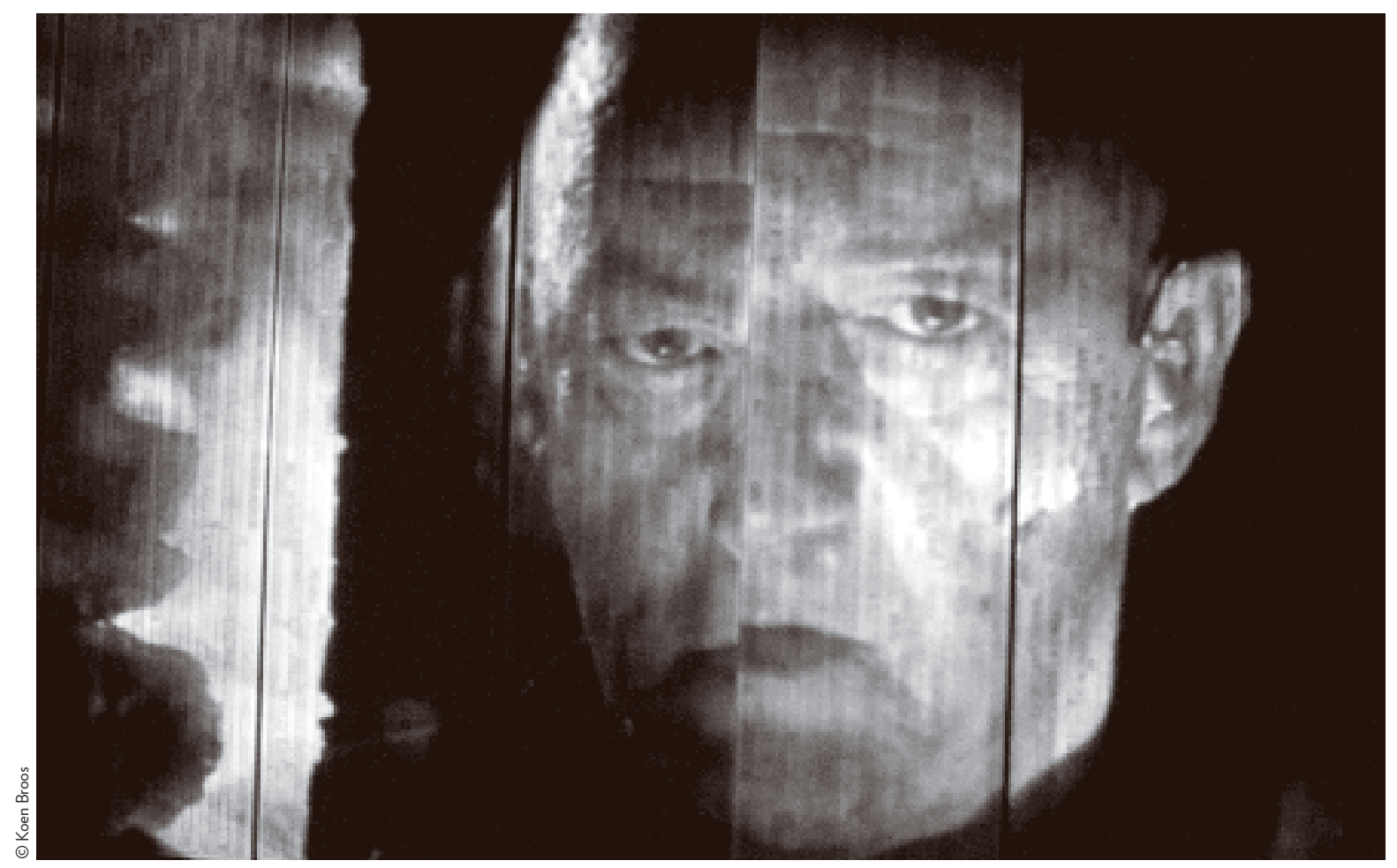

_ Coeur ténébreux.

Littell. L'idée est de montrer comment l'identité fascistoïde est générée aussi par la langue et comment cela influence notre comportement. C'est quelque chose que Littell, dans son essai Le sec et l'humide, analyse dans les écrits de Léon Degrelle, en s'appuyant sur les analyses sociales de Klaus Theweleit. Dans Les Bienveillantes, c'est la même chose, même si Littell ne le dit pas de manière aussi directe. On voit néanmoins comment l'extermination des Juifs pendant la Seconde Guerre mondiale a pu être expliquée, voire justifiée, à travers l'utilisation du langage. Le livre est incroyable en ce qu'il montre comment la justification du meurtre change constamment, comment la manipulation du langage peut venir justifier l'injustifiable, en mettant à distance les gens de leurs émotions. On voit jusqu'où peut aller le pouvoir du langage, comment l'on peut se cacher derrière lui pour commettre des actes que nous savons mauvais, ou pour nous défendre de ceux dont nous sommes pourtant responsables. C'est à travers le langage que tous les responsables se sont défendus des actes qu'ils avaient commis, tout en sachant qu'ils étaient mauvais. Je pense que les faits et détails du passé mentionnés dans Les Bienveillantes font écho à ce qui est en train de se passer aujourd'hui, non pas dans les événements en soi, mais dans les comportements et dans la manière dont on utilise le langage pour se dédouaner de notre responsabilité individuelle. 
Revisiter l'Histoire en déconstruisant les événements (suite)

\section{RÉAFFIRMER LA RESPONSABILITÉ INDIVIDUELLE À TRAVERS LE THÉÂTRE}

Votre manière d'aborder le théâtre pourrait laisser penser que vous faites du théâtre politique. Avez-vous ce sentiment?

G.C. : D’après moi, le théâtre est important pour que le spectateur retrouve sa responsabilité individuelle. Je ne fais pas un théâtre politique en ce sens où je ne souhaite pas imposer aux spectateurs ce que je pense sur tel ou tel sujet. Ce qui m'importe c'est qu'ils forment leur propre opinion, développent leurs propres idées et prennent conscience de leur responsabilité en tant qu'individu. C'est pourquoi je tâche de réunir suffisamment d'informations sans être trop restrictif. La force du théâtre est d'offrir des outils qui permettent de développer cela, et non pas de convaincre qui que ce soit de ce qu'il doit penser, de ce qui est bien ou pas. Le théâtre nécessite du temps et de la distance, du recul. Dans le monde où l'on vit aujourd'hui, prendre deux heures de son temps pour réfléchir à un thème est énorme, notamment pour des jeunes. Notre expérience du temps est en train de changer complètement et le théâtre, en créant une sorte de bulle temporelle, nous offre les moyens de regarder différemment les choses que nous croyons connaître. Aussi, par la fiction nous pouvons, mentalement, nous rapprocher de la réalité. Parce qu’à travers l'imagination que suscite la fiction, nous développons un lien intime, un moyen d'accès très personnel, à quelque chose ou à quelqu'un. Le théâtre repose sur cela. C'est sa force. I 